\title{
\begin{tabular}{l|l} 
Mitraries & DSpace@MIT
\end{tabular}
}

\author{
MIT Open Access Articles
}

\section{Direct and quantitative photothermal absorption spectroscopy of individual particulates}

The MIT Faculty has made this article openly available. Please share how this access benefits you. Your story matters.

Citation: Tong, Jonathan K. et al. “Direct and Quantitative Photothermal Absorption Spectroscopy of Individual Particulates." Applied Physics Letters 103, 26 (December 2013): 261104 (c) 2013 AIP Publishing LLC

As Published: http://dx.doi.org/10.1063/1.4858387

Publisher: American Institute of Physics (AIP)

Persistent URL: http://hdl.handle.net/1721.1/118960

Version: Final published version: final published article, as it appeared in a journal, conference proceedings, or other formally published context

Terms of Use: Article is made available in accordance with the publisher's policy and may be subject to US copyright law. Please refer to the publisher's site for terms of use. 


\title{
Direct and quantitative photothermal absorption spectroscopy of individual particulates
}

\author{
Jonathan K. Tong, ${ }^{1}$ Wei-Chun Hsu, ${ }^{1}$ Sang Eon Han, ${ }^{1, a)}$ Brian R. Burg, ${ }^{1, b)}$ Ruiting Zheng, ${ }^{2}$ \\ Sheng Shen, ${ }^{3}$ and Gang Chen ${ }^{1, c)}$ \\ ${ }^{1}$ Department of Mechanical Engineering, Massachusetts Institute of Technology, Cambridge, \\ Massachusetts 02139, USA \\ ${ }^{2}$ Key Laboratory of Radiation Beam Technology and Materials Modification of Ministry of Education, \\ College of Nuclear Science and Technology, Beijing Normal University, Beijing 100875, \\ People's Republic of China \\ ${ }^{3}$ Department of Mechanical Engineering, Carnegie Mellon University, Pittsburgh, Pennsylvania 15213, USA
}

(Received 4 November 2013; accepted 6 December 2013; published online 23 December 2013)

\begin{abstract}
Photonic structures can exhibit significant absorption enhancement when an object's length scale is comparable to or smaller than the wavelength of light. This property has enabled photonic structures to be an integral component in many applications such as solar cells, light emitting diodes, and photothermal therapy. To characterize this enhancement at the single particulate level, conventional methods have consisted of indirect or qualitative approaches which are often limited to certain sample types. To overcome these limitations, we used a bilayer cantilever to directly and quantitatively measure the spectral absorption efficiency of a single silicon microwire in the visible wavelength range. We demonstrate an absorption enhancement on a per unit volume basis compared to a thin film, which shows good agreement with Mie theory calculations. This approach offers a quantitative approach for broadband absorption measurements on a wide range of photonic structures of different geometric and material compositions. (C) 2013 AIP Publishing LLC. [http://dx.doi.org/10.1063/1.4858387]
\end{abstract}

Photonic structures have provided unparalleled control over the flow of light due to strong light-matter interaction from their sub-wavelength features. In particular, micro/nanoparticulates, such as micro/nanoparticles or micro/nanowires, can exhibit significant absorption enhancement when the length scale is comparable to or smaller than the wavelength of light. This enhancement combined with the inherently low material cost of these structures make them advantageous in applications such as solar cells, light emitting diodes, and photothermal therapy. ${ }^{1-15}$ One of the key challenges in assessing the optical performance of such structures, especially at the single particulate level, is the measurement of the absorption. Conventional broadband spectroscopy techniques typically provide either an indirect or qualitative measure of the absorption efficiency. In this work, we present a direct and quantitative measurement of the spectral absorption efficiency for an individual silicon microwire and demonstrate an absorption enhancement on a per unit volume basis compared to a thin film.

One of the most common techniques used to measure the optical properties of particulates is dark-field spectroscopy. ${ }^{16-19}$ In this method, a white light source is used to illuminate a sample with an oblique hollow light cone, and the scattered light spectra are collected using a spectrometer. In this manner, the scattering properties of particulates are measured. However, the absorptive properties of particles cannot be determined by

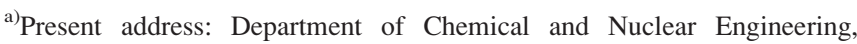
University of New Mexico, Albuquerque, New Mexico 87131, USA.

${ }^{b)}$ Present address: IBM Research-Zürich, 8803 Rüschlikon, Switzerland.

c) Author to whom correspondence should be addressed. Electronic mail: gchen2@mit.edu
}

the scattering properties alone; therefore, absorption cannot be measured with this approach. More recently, photocurrent measurements have been used to qualitatively measure the spectral absorption efficiency of individual semiconductor nanowires. $^{20-23}$ In this method, an Ohmic contact is deposited on one end of the nanowire and a Schottky contact at the other end turning the nanowire into a photodiode. By illuminating the nanowire with light at different wavelengths, the resulting photocurrent can be correlated to the spectral absorption efficiency. In this case, the exact measurement of absorption is hindered by the wavelength-dependent internal quantum efficiency. Studies have also made use of a newer technique known as single particle extinction spectroscopy. ${ }^{24,25}$ Extinction represents the combined effects of scattering and absorption. In the small particle limit where the length scale is typically less than $10 \mathrm{~nm}$, scattering effects become negligible; thus, this method can indirectly measure the spectral absorption efficiency by measuring extinction. However, if scattering effects are appreciable, this approach cannot be used to determine absorption. Currently, no method exists that allows the direct and quantitative measure of spectral absorption efficiency at the single particulate level while also being applicable to a wide range of samples.

In this study, we demonstrate a more widely applicable approach to directly and quantitatively measure the spectral absorption efficiency of individual structures using a bilayer cantilever as a photothermal sensor. ${ }^{26-29}$ These cantilevers consist of two layers of materials with different thermal expansion coefficients. When a temperature change is imposed, the induced thermal stress will cause the cantilever to bend. Since these cantilevers are typically used in atomic force microscopy, their geometry inherently makes them 
highly sensitive thermal sensors. In fact, it has been demonstrated that when lock-in detection is used, these sensors can achieve a temperature resolution of $4 \mu \mathrm{K}$ and a power resolution of $4 \mathrm{pW} .{ }^{30}$ These cantilevers have been used in studies to probe near-field radiative transfer on the order of $100 \mathrm{nW}$, to measure the thermal conductivity of a single polyethylene nanofiber, to map infrared absorption in thin-films with a spatial resolution of $100 \mathrm{~nm}$, and to detect chemical and biological molecules using mid-IR spectroscopy. ${ }^{31-36}$ Because of the high heat flux sensitivity, the use of a bilayer cantilever is well suited for single particle absorption measurements. To demonstrate the feasibility of this approach, we use this technique to measure the spectral absorption efficiency of an individual silicon microwire in the visible wavelength range.

The choice of a silicon microwire was motivated by its current technological importance in applications such as solar photovoltaics and photo detectors. ${ }^{17,22,23}$ To predict the absorptive properties of an individual silicon microwire, the well-established Mie theory was used. ${ }^{37}$ In this formalism, analytical solutions to the Maxwell equations are obtained for the case of a monochromatic plane wave incident on an infinitely long cylinder. ${ }^{38}$ The spectral absorption efficiency, $\mathrm{Q}_{\mathrm{abs}}$, can be defined as follows: ${ }^{38}$

$$
\mathrm{Q}_{\mathrm{abs}}(\lambda)=\frac{\mathrm{P}_{\mathrm{abs}}(\lambda)}{\mathrm{P}_{\mathrm{inc}}(\lambda)},
$$

where $\lambda$ is the wavelength, $\mathrm{P}_{\mathrm{abs}}(\lambda)$ is the absorbed power, and $P_{\text {inc }}(\lambda)$ is the power geometrically incident on the cross section of the object. Using this formalism, the spectral absorption efficiency was calculated for a silicon microwire as shown in Fig. 1(a). The incoming light was assumed to be unpolarized and at normal incidence. The optical constants of intrinsic monocrystalline silicon were used for the calculation. ${ }^{39}$

To assess the enhancement in absorption, we can compare the spectral absorption efficiency to a volumetrically equivalent thin film. Since absorption is dictated by the particulates' cross section, we can consider an equivalent cross section of a thin film whose width is equal to the diameter of a wire. For the thin film to be volumetrically equivalent, the thickness must equal to $t=\pi D / 4$. Using the Fresnel coefficients, the absorptance of a thin film was calculated as shown in Fig. 1(a). By taking the ratio of the spectral absorption efficiency of the microwire to the corresponding volumetrically equivalent thin film, an absorption enhancement, R, was obtained as shown in Fig. 1(b). For a diameter $\mathrm{D}=1000 \mathrm{~nm}$, the absorption enhancement, as shown in Fig. 1(b), is higher overall compared to the thin film with a peak value of $R=2.621$ at a wavelength of $592 \mathrm{~nm}$ in the wavelength range considered.

To measure the absorption enhancement of a single silicon microwire, we used a bilayer cantilever to directly measure the absorbed power by the microwire when under illumination from a monochromatic light source. This was accomplished by attaching the microwire directly to the cantilever. Light absorbed by the microwire conducted as heat to the cantilever resulting in a temperature change. The thermal expansion mismatch then caused the bilayer cantilever to bend. Therefore, the bending response of the cantilever was directly correlated to the absorbed power. Following a series of calibrations, we were then able to extract quantitatively the absorption efficiency from the bending response of the bilayer cantilever.

A pyrex nitride gold coated tipless cantilever (NanoWorld, PNP-TR-TL-Au) was used in this work. A silicon microwire is suspended from one end in order to avoid illumination of the cantilever during measurement. As a result, the microwire in this configuration also functions as a pathway for heat to conduct to the cantilever.

In this study, n-type silicon microwires were fabricated using a metal-assisted etching technique. ${ }^{40,41}$ A SEM cross section image of a silicon microwire sample is shown in Fig. 2(a). These nano/microwires typically had a length of $100 \mu \mathrm{m}$ and a diameter that ranged from 100 to $1000 \mathrm{~nm}$. To attach the microwire to the cantilever, a set of piezoelectric nanomanipulators were used in a dual-column focused ion beam system. An ultrahigh vacuum adhesive (Kleindiek, SemGlu) was applied to glue the microwire to the cantilever. Figure 2(b) shows an image of the silicon microwire used for measurement attached to the cantilever. For this particular microwire, the average diameter was $983 \mathrm{~nm}$ and the length was $92.7 \mu \mathrm{m}$.

A schematic of the measurement platform is shown in Fig. 2(c). This platform is based on the previous studies. ${ }^{26-29}$ The cantilever was mounted onto a copper plate using silver epoxy. The copper plate served as both a heat reservoir and an electrical conductor in order to electrically ground the sample. To measure the bending response, a $635 \mathrm{~nm}$ continuous-wave $(\mathrm{CW})$ laser is reflected off the tip of the cantilever onto a position sensitive detector (PSD). A $670 \mathrm{~nm}$ transistor-transistor-logic (TTL) modulated laser was (a)

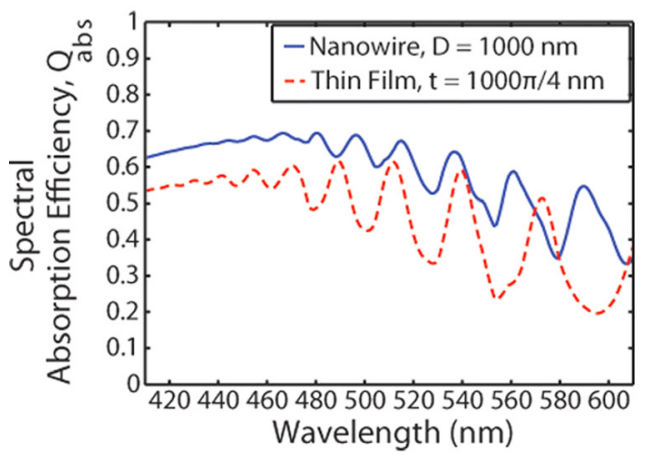

(b)

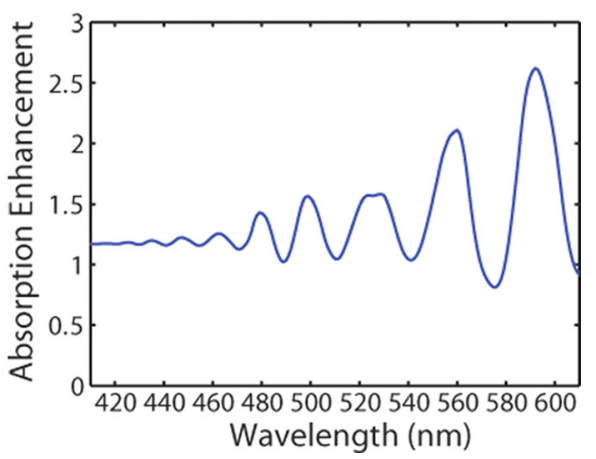

FIG. 1. Theoretical predictions of absorption enhancement for an intrinsic monocrystalline silicon microwire. (a) The spectral absorption efficiency at a fixed diameter of $\mathrm{D}=1000 \mathrm{~nm}$. The spectral absorptance of a volumetrically equivalent silicon thin film is also plotted. (b) The absorption enhancement obtained by taking the ratio of the spectral absorption efficiency of the microwire to the corresponding thin film. 
(a)

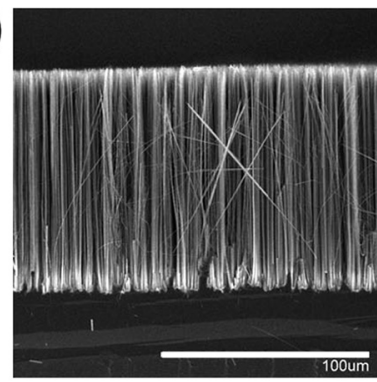

(b)

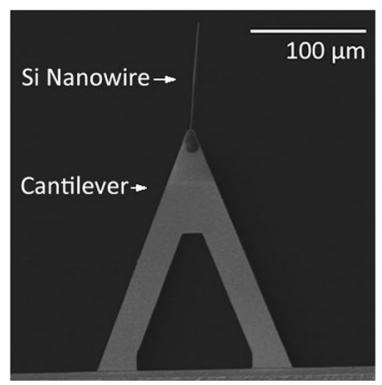

(c)

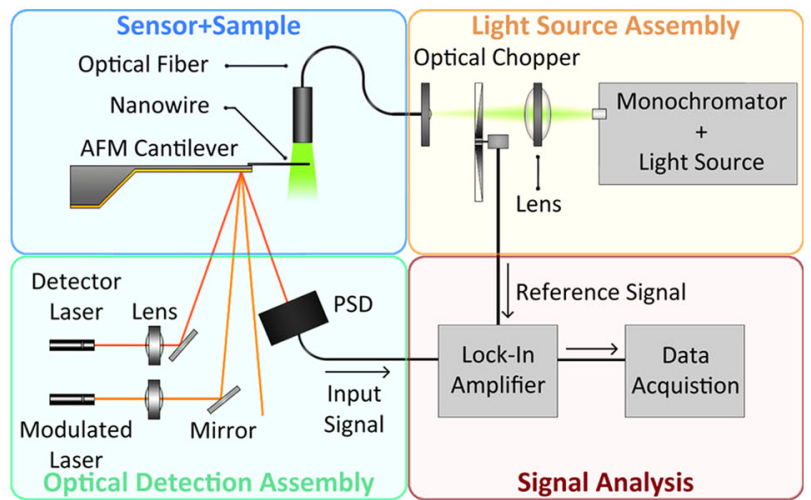

FIG. 2. Sample preparation and measurement system using a bilayer cantilever. (a) A cross section image, taken with a SEM, of the silicon nano/microwire arrays synthesized using metal-assisted etching. (b) A SEM image of a silicon microwire $(\mathrm{D}=983 \mathrm{~nm}, \mathrm{~L}=92.7 \mu \mathrm{m})$ attached to a bilayer cantilever using adhesive and piezoelectric nanomanipulators. (c) A schematic diagram of the measurement platform. The "Optical Detection Assembly" consists of components to optically measure the bending response of the cantilever such as lasers, optics, and the PSD. The "Sensor + Sample" consist of the bilayer cantilever and the attached sample. The "Light Source Assembly" consists of a monochromator and xenon lamp source coupled to an optical fiber which directs light onto the sample. The "Signal Analysis" consists of a lock-in amplifier to enhance the sensitivity of the system and data acquisition instruments.

also incorporated for the sole purpose of measuring a frequency response correction factor as will be discussed. These components were mounted onto an optical breadboard and placed directly into a vacuum chamber to eliminate heat losses via convection. The breadboard was supported by rubber dampers to reduce vibrational noise. A xenon lamp source and a monochromator provided visible wavelength light. The light was coupled to a multimode optical fiber and routed through an optical feedthrough into the vacuum chamber. Inside the vacuum chamber, a cleaved optical fiber then directed the light onto the sample. An optical chopper is used to modulate the light input for lock-in detection. A data acquisition system recorded the bending response from the PSD.

The choice of the modulation frequency was dictated by the thermal time constant of the cantilever and the silicon microwire. Based on the geometry and properties of the cantilever, the time constant was estimated to be $\tau \approx 0.6 \mathrm{~ms}$, similar to the literature values. ${ }^{27-29}$ The cut-off frequency was therefore $\mathrm{f}=1 /(2 \pi \tau) \approx 265 \mathrm{~Hz}$. For the silicon microwire, a thermal conductivity of $\mathrm{k} \approx 10 \mathrm{~W} \mathrm{~m}^{-1} \mathrm{~K}^{-1}$ was used as a conservative estimate to account for size effects. ${ }^{42}$ This value corresponds to a microwire with a diameter of $150 \mathrm{~nm}$. The time constant is $\tau \approx 1 \mathrm{~ms}$ and the cut-off frequency is $\mathrm{f} \approx 160 \mathrm{~Hz}$, indicating the silicon microwire is frequency limiting. ${ }^{43}$ It was also observed that vibration, thermal drift, and electrical noise were significant below $100 \mathrm{~Hz}$. In addition, integer multiples of the power line frequency at $60 \mathrm{~Hz}$ were avoided. Based on these limits, a modulation frequency of $140 \mathrm{~Hz}$ was used.

To quantitatively measure the spectral absorption efficiency, several calibration measurements were required in order to extract the absorption efficiency from the bending response of the cantilever. Equation (1) can be expanded in terms of these calibration parameters

$$
\mathrm{Q}_{\mathrm{abs}}(\lambda)=\frac{\mathrm{P}_{\mathrm{abs}}(\lambda)}{\mathrm{P}_{\mathrm{inc}}(\lambda)}=\frac{\left[\mathrm{X}(\lambda) /\left(\beta \mathrm{S}_{\mathrm{p}}\right)\right]}{\left[\mathrm{P}_{\mathrm{tot}}(\lambda) \mathrm{f}_{\mathrm{inc}}\right]},
$$

where $\mathrm{X}(\lambda)$ is the spectral bending response of the cantilever due to the sample, $\beta$ is a frequency response correction factor, $\mathrm{S}_{\mathrm{p}}$ is the power sensitivity, $\mathrm{P}_{\text {tot }}(\lambda)$ is the total power from the optical fiber, and $f_{\text {inc }}$ is the fraction of the input power that is incident on the sample. ${ }^{44}$

The spectral bending response, $\mathrm{X}(\lambda)$, was measured by illuminating the silicon microwire with monochromatic light in the wavelength range of 420 to $600 \mathrm{~nm}$ in increments of $10 \mathrm{~nm}$. Lock-in detection was required to provide the necessary sensitivity in this system. However, a periodic heat input resulted in a frequency dependent bending response by virtue of a frequency dependent thermal response. In general, a higher modulation frequency results in attenuation of the bending response. To account for this effect, a frequency response correction factor, $\beta$, was measured by using the TTL modulated laser to compare the bending amplitude under periodic heating and steady heating. The attenuation factor was measured to be $\beta=0.4032$.

In order to extract quantitative data from the bending response, a power calibration was required. ${ }^{45}$ This calibration correlates the bending response to a known heat input in order to obtain a power sensitivity, $\mathrm{S}_{\mathrm{p}}$. This power sensitivity can then be used to extract an absorbed power at a given wavelength from the bending response. For this calibration, the power of the $\mathrm{CW}$ detector laser was varied by changing the input voltage and the bending response was subsequently recorded. The measured power sensitivity was $\left|\mathrm{S}_{\mathrm{p}}\right|=103.9 \mathrm{~V} / \mathrm{mW}$.

The total output power from the optical fiber, $\mathrm{P}_{\text {tot }}(\lambda)$, was measured using a photodiode. To measure the fraction of light incident on the sample, $f_{\text {inc }}$, the position of the sample within the beam spot must be precisely known. Furthermore, the beam intensity exhibited a Gaussian distribution. To account for this Gaussian distribution, an area integral was performed over the beam spot cross section and the illuminated cross section of the microwire. By taking a ratio of these two quantities, the fraction was calculated to be $\mathrm{f}_{\text {inc }}=9.83 \times 10^{-4}$.

From these calibrations, the absorbed power of the silicon microwire ranged from $352 \mathrm{pW}$ at a wavelength of $600 \mathrm{~nm}$ to $1.08 \mathrm{nW}$ at a wavelength of $470 \mathrm{~nm}$. The incident power on the microwire ranged from $856 \mathrm{pW}$ at a wavelength of $600 \mathrm{~nm}$ to $1.589 \mathrm{nW}$ at a wavelength of $470 \mathrm{~nm}$. The spectral absorption efficiency of the silicon microwire is shown in Fig. 3. The error bars represent a 95\% confidence 


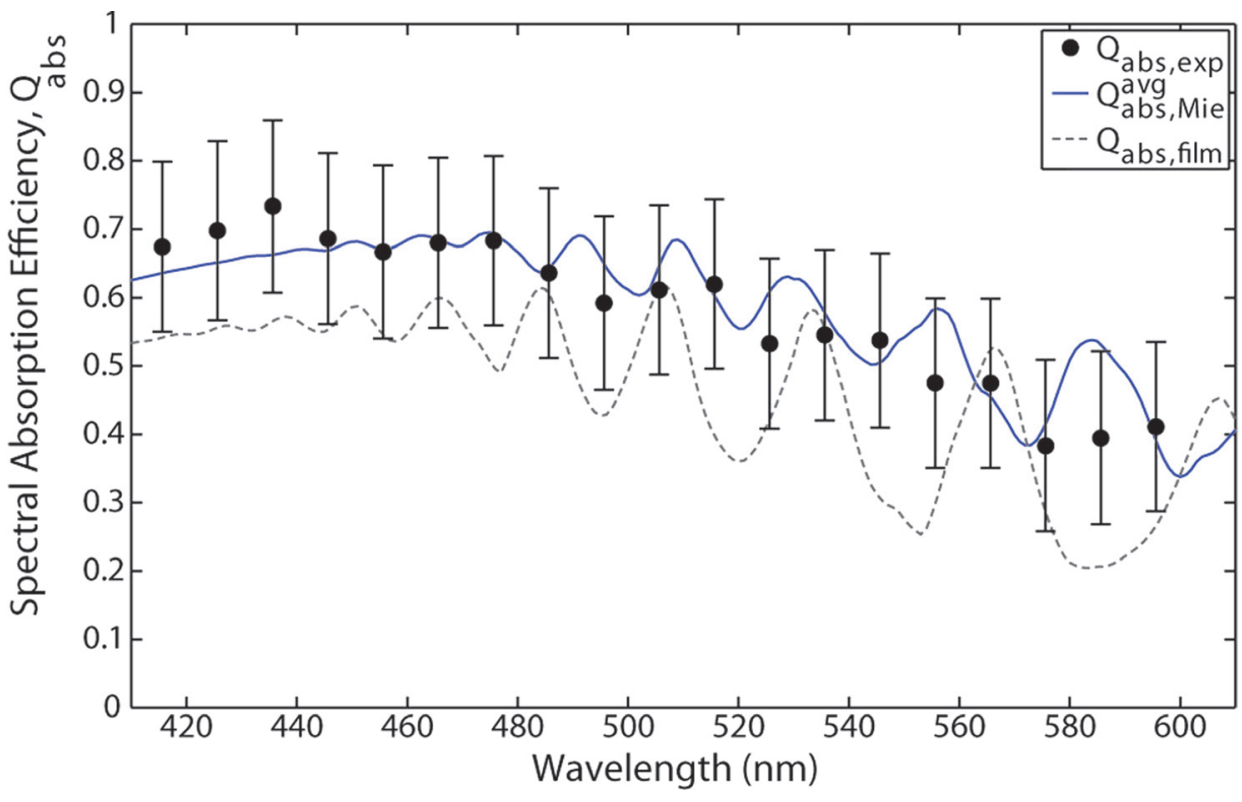

FIG. 3. The spectral absorption efficiency, $\mathrm{Q}_{\text {abs,exp }}$, measured for an n-type silicon microwire from the spectral bending response and the calibrations from Eq. (2). The error bars represent a 95\% confidence interval. The theoretical spectral absorption efficiency obtained from Mie theory, $\mathrm{Q}_{\text {abs, Mie }}^{\text {avg }}$, is also plotted for a uniform microwire with an average diameter of $\mathrm{D}=983 \mathrm{~nm}$. For further comparison, the theoretical spectral absorption efficiency for a volumetrically equivalent $(t=983 \pi / 4 \mathrm{~nm})$ thin film, Qabs,film, is also shown. interval with an average uncertainty of $12.5 \%$. This uncertainty is primarily due to the high sensitivity of the parameter, $f_{\text {inc }}$, to the position of the microwire and the measurement of absorbed power for the power calibration. The theoretical spectral absorption efficiency for a uniform microwire with an average diameter of $983 \mathrm{~nm}$ and a volumetrically equivalent thin film are also shown in Fig. 3. Compared to the theory, the experimental results exhibit moderate agreement with an average percent error of 9.2\% . The maximum percent error was found to be $24.6 \%$ at a wavelength of $580 \mathrm{~nm}$.

To assess the significance of the discrepancy between Mie theory and our experiment, several corrections were applied to the theoretical results to better coincide with experimental conditions. First, a size average was performed to account for non-uniformity in the microwire. The standard deviation of this non-uniformity was $28 \mathrm{~nm}$ for the section of the microwire illuminated. As a simple approximation, a weighted average was applied to the spectral absorption efficiency by superimposing theoretical solutions calculated at different diameters as measured at different points in the SEM image. Second, a wavelength average was performed to account for the finite bandwidth of the incoming light. The full-width half-maximum was measured to be $7-8 \mathrm{~nm}$ for all wavelengths. As a simple approximation, a linear fit is applied to the light input and used as a weighting function. In addition, the angle of incidence, taken perpendicular to the axis of the microwire, and the polarization of the incoming light were also investigated and determined to be approximately normally incident and unpolarized, respectively.

These corrections were applied to the theoretical results as shown in Fig. 4(a). It can be observed that size averaging smeared the interference peaks resulting in smaller amplitude oscillations. The addition of wavelength averaging did not alter the results significantly. Overall, the experimental results exhibit a much better agreement with the modified theoretical curve. The average percent error decreased to $4.7 \%$. The maximum percent error also decreased to $14.2 \%$ at $580 \mathrm{~nm}$. The absorption enhancement was obtained by normalizing the experimental results to the theoretical spectral absorptance for a volumetrically equivalent thin film with a thickness of $t=983 \pi / 4 \mathrm{~nm}$. The results are shown in Fig. 4(b). The experimental results indicate an overall
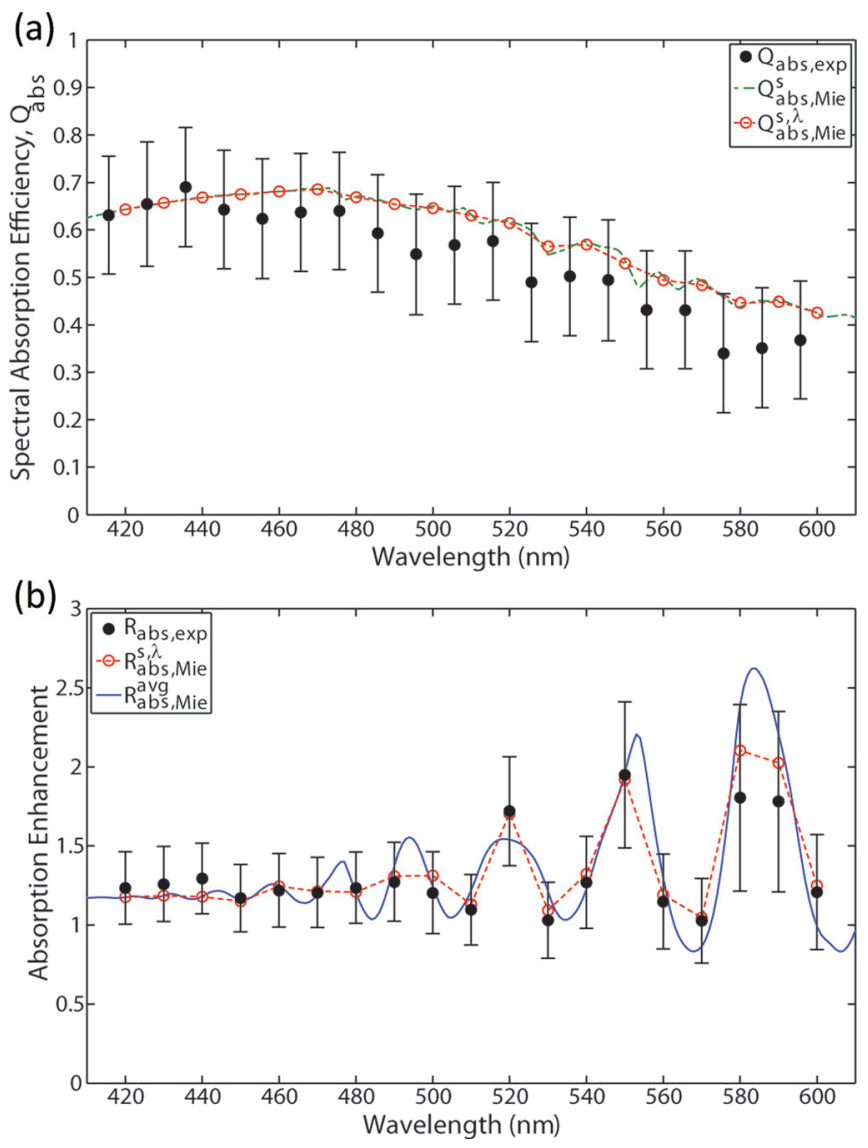

FIG. 4. Measured absorption enhancement and comparisons with theory. (a) The experimentally measured absorption efficiency, $Q_{\text {abs,exp }}$, compared to a size corrected theoretical curve, $\mathrm{Q}_{\mathrm{abs}, \text { Mie }}^{\mathrm{s}}$ and a size and wavelength corrected theoretical curve, $\mathrm{Q}_{\mathrm{abs}, \mathrm{sie}}^{\mathrm{s}, \lambda}$ (b) The absorption enhancement for the experimental results, $R_{\text {abs,exp }}$, the corrected Mie theory results, $R_{\text {abs, Mie }}^{\mathrm{s}, \lambda}$, and the Mie theory results for a uniform microwire, $R_{a b s, M i e}^{\text {avg }}$, obtained by normalizing to the spectral absorptance of a volumetrically equivalent thin film. 
absorption enhancement compared to the thin film across the measured wavelength range.

In summary, we utilized a bilayer cantilever as a photothermal sensor to directly and quantitatively measure the spectral absorption efficiency of a single silicon microwire. We demonstrated an absorption enhancement on a per unit volume basis compared to a thin film, which exhibited good agreement with theoretical predictions. We envision the excellent sensitivity and broad applicability of the method developed in this work can be applied to the measurement of nanoparticulates and photonic nanostructures with different geometric and material compositions. Recent studies have also introduced greater functionality to the cantilever platform and absolute absorption measurements in the infrared wavelength range. ${ }^{46,47}$ The approach developed in this work therefore has the potential to enable broadband absorption measurements in the optical wavelength range to an extent previously unattainable.

The authors would like to thank D. Kraemer for access to the vacuum chamber system, Dr. G. McMahon for his assistance with the sample attachment; P. Sambegoro, Dr. A. Mavrokefalos, Dr. A. Minnich, K. Collins, M. Luckyanova, and L. Zeng for helpful discussions. Sample attachment via focused ion beam system was carried out at Boston College's Integrated Science Cleanroom and Nanofabrication Facility. This work was supported by DOE Grant No. DE-FG0202ER45977 (J.K.T., S.S., and G.C.), AFOSR MURI from UIUC (W.-C.H. and S.E.H.), and by the National Research Fund, Luxembourg, cofunded under Marie Curie Actions of the European Commission (FP7-COFUND 893874) (B.R.B.).

${ }^{1}$ M. D. Kelzenberg, S. W. Boettcher, J. A. Petykiewicz, D. B. TurnerEvans, M. C. Putnam, E. L. Warren, J. M. Spurgeon, R. M. Briggs, N. S. Lewis, and H. A. Atwater, Nature Mater. 9(3), 239-244 (2010).

${ }^{2}$ R. Bardhan, S. Lal, A. Joshi, and N. J. Halas, Acc. Chem. Res. 44(10), 936-946 (2011).

${ }^{3}$ S. A. Mann and E. C. Garnett, Nano Lett. 13(7), 3173-3178 (2013).

${ }^{4}$ P. Krogstrup, H. I. Jorgensen, M. Heiss, O. Demichel, J. V. Holm, M. Aagesen, J. Nygard, and A. Fontcuberta i Morral, Nat. Photonics 7(4), 306-310 (2013).

${ }^{5}$ L. Hu and G. Chen, Nano Lett. 7(11), 3249-3252 (2007).

${ }^{6}$ J. B. Baxter and E. S. Aydil, Appl. Phys. Lett. 86(5), 053114 (2005).

${ }^{7}$ L. Tsakalakos, J. Balch, J. Fronheiser, M.-Y. Shih, S. F. LeBoeuf, M. Pietrzykowski, P. J. Codella, B. A. Korevaar, O. V. Sulima, J. Rand, A. Davuluru, and U. Rapol, J. Nanophotonics 1, 013552 (2007).

${ }^{8}$ E. C. Garnett and P. Yang, J. Am. Chem. Soc. 130(29), 9224-9225 (2008).

${ }^{9}$ B. Tian, T. J. Kempa, and C. M. Lieber, Chem. Soc. Rev. 38(1), 16-24 (2009).

${ }^{10}$ H. Ningfeng, L. Chenxi, and M. L. Povinelli, J. Opt. 14(2), 024004 (2012).

${ }^{11}$ M. C. McAlpine, R. S. Friedman, S. Jin, K.-h. Lin, W. U. Wang, and C. M. Lieber, Nano Lett. 3(11), 1531-1535 (2003).

${ }^{12}$ E. Hutter and J. H. Fendler, Adv. Mater. 16(19), 1685-1706 (2004).

${ }^{13}$ Y. Huang, X. Duan, and C. M. Lieber, Small 1(1), 142-147 (2005).

${ }^{14}$ P. K. Jain, X. Huang, I. H. El-Sayed, and M. A. El-Sayed, Acc. Chem. Res. 41(12), 1578-1586 (2008).

${ }^{15}$ H. A. Atwater and A. Polman, Nature Mater. 9(3), 205-213 (2010).
${ }^{16}$ C. Sönnichsen, T. Franzl, T. Wilk, G. von Plessen, J. Feldmann, O. Wilson, and P. Mulvaney, Phys. Rev. Lett. 88(7), 077402 (2002).

${ }^{17}$ G. Brönstrup, N. Jahr, C. Leiterer, A. Csáki, W. Fritzsche, and S. Christiansen, ACS Nano 4(12), 7113-7122 (2010).

${ }^{18}$ C. L. Nehl, N. K. Grady, G. P. Goodrich, F. Tam, N. J. Halas, and J. H. Hafner, Nano Lett. 4(12), 2355-2359 (2004).

${ }^{19}$ C. L. Nehl, H. Liao, and J. H. Hafner, Nano Lett. 6(4), 683-688 (2006).

${ }^{20}$ L. Cao, J. S. White, J.-S. Park, J. A. Schuller, B. M. Clemens, and M. L. Brongersma, Nature Mater. 8(8), 643-647 (2009).

${ }^{21}$ L. Cao, J.-S. Park, P. Fan, B. Clemens, and M. L. Brongersma, Nano Lett. 10(4), 1229-1233 (2010).

${ }^{22}$ L. Cao, P. Fan, A. P. Vasudev, J. S. White, Z. Yu, W. Cai, J. A. Schuller, S. Fan, and M. L. Brongersma, Nano Lett. 10(2), 439-445 (2010).

${ }^{23}$ T. Xu, Y. Lambert, C. Krzeminski, B. Grandidier, D. Stievenard, G. Leveque, A. Akjouj, Y. Pennec, and B. Djafari-Rouhani, J. Appl. Phys. 112(3), 033506 (2012).

${ }^{24}$ J. Giblin, F. Vietmeyer, M. P. McDonald, and M. Kuno, Nano Lett. 11(8), 3307-3311 (2011).

${ }^{25}$ F. Vietmeyer, M. P. McDonald, and M. Kuno, J. Phys. Chem. C 116(23), 12379-12396 (2012).

${ }^{26}$ J. R. Barnes, R. J. Stephenson, M. E. Welland, C. Gerber, and J. K. Gimzewski, Nature 372(6501), 79-81 (1994).

${ }^{27}$ J. R. Barnes, R. J. Stephenson, C. N. Woodburn, S. J. O'Shea, M. E. Welland, T. Rayment, J. K. Gimzewski, and C. Gerber, Rev. Sci. Instrum. 65(12), 3793-3798 (1994).

${ }^{28}$ J. Lai, T. Perazzo, Z. Shi, and A. Majumdar, Sens. Actuators, A 58(2), 113-119 (1997).

${ }^{29}$ J. Varesi, J. Lai, T. Perazzo, Z. Shi, and A. Majumdar, Appl. Phys. Lett. 71(3), 306-308 (1997).

${ }^{30}$ S. Sadat, Y. J. Chua, W. Lee, Y. Ganjeh, K. Kurabayashi, E. Meyhofer, and P. Reddy, Appl. Phys. Lett. 99(4), 043106 (2011).

${ }^{31}$ S. Shen, A. Narayanaswamy, and G. Chen, Nano Lett. 9(8), 2909-2913 (2009).

${ }^{32}$ S. Shen, A. Henry, J. Tong, R. Zheng, and G. Chen, Nat. Nanotechnol 5(4), 251-255 (2010).

${ }^{33}$ A. Wig, A. Passian, E. Arakawa, T. L. Ferrell, and T. Thundat, J. Appl. Phys. 95(3), 1162-1165 (2004).

${ }^{34}$ K. Kjoller, J. R. Felts, D. Cook, C. B. Prater, and W. P. King, Nanotechnology 21(18), 185705 (2010).

${ }^{35}$ B. Kwon, W. P. King, M. Schulmerich, and R. Bhargava, in Conference on IEEE Sensors (2010), pp. 2405-2408.

${ }^{36}$ L. Tetard, A. Passian, R. H. Farahi, B. H. Davison, and T. Thundat, Opt. Lett. 36(16), 3251-3253 (2011).

${ }^{37}$ C. F. Bohren, Am. J. Phys. 51(4), 323-327 (1983).

${ }^{38}$ C. Bohren and D. Huffman, Absorption and Scattering of Light by Small Particles, Wiley Science Paperback Series (Wiley-VCH, 1998).

${ }^{39}$ D. F. Edwards, "Silicon (Si)" in Handbook of Optical Constants of Solids, edited by E. D. Palik (Academic Press, 1985), Chap. 2.2, pp. 547-569.

${ }^{40}$ K. Peng, Y. Yan, S. Gao, and J. Zhu, Adv. Funct. Mater. 13(2), 127-132 (2003).

${ }^{41}$ K. Peng, Y. Wu, H. Fang, X. Zhong, Y. Xu, and J. Zhu, Angew. Chem., Int. Ed. 44(18), 2737-2742 (2005).

${ }^{42}$ D. Li, Y. Wu, P. Kim, L. Shi, P. Yang, and A. Majumdar, Appl. Phys. Lett. 83(14), 2934-2936 (2003).

${ }^{43}$ A. I. Hochbaum, R. Chen, R. D. Delgado, W. Liang, E. C. Garnett, M. Najarian, A. Majumdar, and P. Yang, Nature 451(7175), 163-167 (2008).

${ }^{44}$ See supplementary material at http://dx.doi.org/10.1063/1.4858387 for more details of each calibration procedure.

${ }^{45}$ S. Shen, A. Narayanaswamy, S. Goh, and G. Chen, Appl. Phys. Lett. 92(6), 063509 (2008).

${ }^{46}$ B. R. Burg, J. K. Tong, W.-C. Hsu, and G. Chen, Rev. Sci. Instrum. 83(10), 104902 (2012).

${ }^{47}$ W.-C. Hsu, J. K. Tong, B. Liao, B. R. Burg, and G. Chen, Appl. Phys. Lett. 102(5), 051901 (2013). 